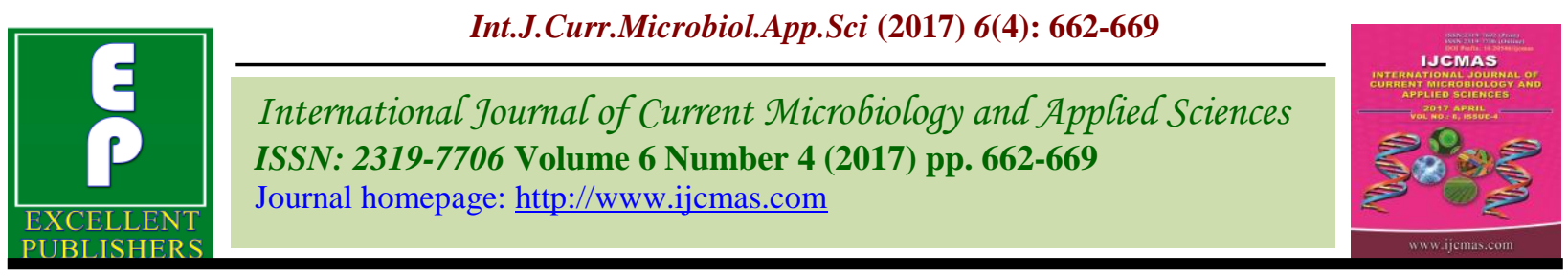

Original Research Article https://doi.org/10.20546/ijcmas.2017.604.081

\title{
Incidence of Catheter Associated Urinary Tract Infection in Medical ICU in a Tertiary Care Hospital
}

\author{
V. Sangamithra*, Sneka, Shabana Praveen and Manonmoney \\ Department of Microbiology, SRM Medical College and Research Institute, Chennai, India \\ *Corresponding author
}

\author{
A B S T R A C T
}

\begin{tabular}{|l|}
\hline Ke y w or d s \\
Indwelling devices, \\
nosocomial \\
infections, \\
Catheter, UTI. \\
\hline Article Info \\
\hline $\begin{array}{l}\text { Accepted: } \\
\text { 06 March } 2017 \\
\text { Available Online: } \\
\text { 10 April } 2017\end{array}$ \\
\hline \hline
\end{tabular}

The Centers for Disease Control and Prevention (CDC) defines CAUTI for those patients who have an indwelling catheter in place for $48 \mathrm{~h}$ or more and symptoms such as fever or chills, new onset of burning pain, urgency or frequency if not catheterized at that point of time, change in urine character, flank or suprapubic pain or tenderness or change or decrease in mental or functional status in patients. CAUTI is usually presence of at least $10 \hat{\mathrm{A}}^{3}$ colony-forming units $(\mathrm{cfu}) / \mathrm{mL}$ of 1 or 2 micro-organisms by urine culture. In Indian population, catheter-associated urinary tract infection (CAUTI) is an important cause of morbidity and mortality, affecting all ages. The study is aimed to find the microbial pathogens \& their antibiotic susceptibility of catheterised patients in Intensive care unit of SRM Medical college hospital. A total of 196 non-repetitive catheterised urine samples taken aseptically from patients admitted in the ICU from October 2016 to December 2016.The demographic profile showed 128 (65\%) males and 68 (35\%) female. E. coli was $25(36 \%)$ the commonest followed by Enterococcus spp. 17 (25\%), Klebsiella species 14(20\%) \& Pseudomonas spp. 4 (5\%).

\section{Introduction}

Indwelling intravascular and urinary catheters are essential components of modern medical care. Unfortunately, indwelling devices significantly increase the risk of iatrogenic infection, particularly in an already fragile patient population. Most nosocomial infections in severely ill patients are associated with the very medical devices that provide life-sustaining care (Ihnsook Jeong et al., 2010).

Urinary tract infections are the most common type of healthcare-associated infection, accounting for more than $30 \%$ of infections reported by acute care hospitals. Virtually all healthcare-associated UTIs are caused by instrumentation of the urinary tract. Catheterassociated urinary tract infection (CAUTI) has been associated with increased morbidity, mortality, hospital cost and length of stay. In addition, hospital acquired CAUTIs are often due to multidrug resistant strains which require higher antibiotics and these strains may spread to other patients (Bagchi et al., 2015).

The mortality rate of catheter associated UTI is less than 5\%. However, since the number of bladder catheters inserted each year is more than 30 million, at least 6 times higher than the number of central venous catheters, catheter-associated UTI is the second most 
common cause of nosocomial bloodstream infection (Ihnsook Jeong et al., 2010).

\section{Routes of infection 1}

At the time of catheter insertion where organisms may be pushed into the previously uninfected bladder.

Extra luminal colonization of the catheter with ascension of organisms into the urinary tract.

Intraluminal colonization of the catheter with ascension of microorganisms (Closed systems are designed to minimize intraluminal infection by preventing exogenous contamination). Acquisition of the infection via the lymphatic or haematogenous route is a proven, though minor portal of entry.

Within 8 hours of insertion of a catheter, a biofilm can be found on the surface of the catheter, drainage bag and mucosa consisting of Tamm-horsefall protein, struvite and apatite crystals, bacterial polysaccharides, glycocalyces and living bacteria and is composed of three layers. Organisms within the biofilm are well protected from mechanical flushing by urine flow, other host defenses and antibiotic.

The duration of catheterisation is directly related to the development of bacteriuria. The overall incidence of nosocomial urinary tract infection among these patients is 35 to $10 \%$ (average 5\%) per day. Bacteremia is attributed to the urinary tract.

Patients who develop a nosocomial infection have their hospital stay extended by approximately 3 days and nearly 3 times more likely to die during hospitalisation than patients without such infections. The case fatality rate from UTI related nosocomial bacteraemia is approximately $13 \%$ severely ill patients at highest risk.
Catheter-Associated Urinary tract Infection (CAUTI) can lead to complications such as cystitis, pyelonephritis, Gram-negative bacteremia, prostatitis, epididymitis and orchitis in males and less commonly, endocarditis, vertebral osteomyelitis, septic arthritis, endophthalmitis, and meningitis in all patients. Complications associated causes discomfort to the patient, prolonged hospital stay and increased cost and mortality.

Prevention of nosocomial UTI and its complications can reduce the rate of morbidity and mortality and expenses secondary to infection. It is hoped that medical technology will allow the advancement in catheterization procedures, need and duration for catheterization and provide advance improvements in the design of drainage system of urinary catheter. Biomaterial research is an exploding new science, and research must continue with these new materials in respect to mucosal biocompatibility and effectiveness in reducing bacterial biofilm attachment. It is anticipated that new biomaterials will eventually reduce bacterial adherence and biofilm formation and subsequently decrease the rate of catheterassociated infection. New antibiotics being developed may be able to penetrate the bacterial biofilm and may be more effective in this and other prosthesis-related infections. Further studies are required to rationalize the use of antibiotics both to prevent and treat catheter-associated infection. For now the most effective way to reduce the incidence of catheter-associated infection is to avoid indwelling Foley catheterization if at all possible, or at least to reduce the length of time the catheter remains in the bladder.

\section{Materials and Methods}

The study was carried in Department of Microbiology of SRM Medical college hospital a teaching tertiary care hospital. A total of 196 consecutive non repetitive urine 
samples of patients with indwelling urethral catheter inserted under aseptic conditions for various medical conditions were included in the study. Samples were collected over a period of 3 months from patients admitted to the ICU.

\section{Specimen collection}

Prior to catheter change or removal from each patient, urine sample were collected aseptically using a sterile needle and syringe from the distal edge of catheter tube into the sterile universal container and transported to the microbiology laboratory for analysis with minimum delay. Patient with symptoms of UTI prior to the catheterization and paediatric patients were excluded.

\section{Microbiology}

The samples were processed by the routine standard laboratory procedure. This included microscopy, culture identification and antibiotic susceptibility testing. Urine microscopy was performed on centrifuged catheter urine specimen. Culture was set up on Cysteine Lactose Electrolyte Deficient Agar for isolating all kind of urinary pathogens; in few cases we used blood agar and MacConkey agar.

Semiquantitative method of urine culture was followed. A sterile calibrated wire loop was used to deliver a loopful $(0.01 \mathrm{ml})$ of urine onto each culture media.

All the culture plates were incubated at $37^{\circ} \mathrm{C}$ aerobically for $18-24 \mathrm{~h}$. All the culture positive isolates were identified by their colony morphology and gram stain and characterized biochemically for species identification. Isolate suggestive of the yeast were subcultured on Sabouraud's dextrose agar. Further identification was done by demonstration of germ tube. Antimicrobial susceptibility testing was done by Kirby
Bauer disk-diffusion method on MullerHinton agar.

\section{Results and Discussion}

A total 196 urine sample from catheterized patients were obtained. Of the total 196 patients $128(65 \%)$ were male and $68(35 \%)$ were female patients (Figures 1 and 2).

Coming to microscopic examination, out of 196urine samples, 91 samples showed the evidence of pus cells and or micro-organisms.

Following the culture of 196 urine samples from catheterized patients 105 (54\%) was sterile and 91 samples showed growth of which $69(76 \%)$ were bacterial and 22(24\%) showed fungal growth (Figure 3 ).

Off the 69 bacterial isolates, E. coli was isolated in $25(36 \%)$ cases and found to be most common. This was, followed by the Enterococcus spp. in $17(25 \%)$ cases, Klebsiella species in 14 (20\%) and Pseudomonas spp. in 4 (5\%) cases.

Other Gram-negative bacteria such as Proteus mirabilis, Proteus vulgaris, Acinetobacter were found in $3 \%, 1 \%$ and $4 \%$ cases. Grampositive bacteria like Staphylococcus aureus and Methicillin-Resistant Staphylococcus aureus were identified in $1(1 \%)$ cases each (Figure 4).

Coming to the fungal isolates, $22(24 \%)$ of the cases showing fungal yeast growth. Candida spp was the most common. Non albicans Candida 19(86\%) isolated more commonly than Candida albicans 3(14\%) (Figure 5).

Table 1 shows that the incidence of UTI was significantly higher as the days of catheterization increased. In vitro, antibiotic susceptibility pattern of Gram-negative and Gram-positive organism found that there is high resistance to commonly used antibiotics for both Gram-positive and Gram negative 
organisms, however Imipenem (95\% and tazobactam (82\% sensitivity) appears to sensitivity) and combination of ceftazidime be effective.

Table.1 Days of catheterisation and occurrence of UTI

\begin{tabular}{|c|c|}
\hline No: of days & Presence of UTI $(\%)$ \\
\hline $1-3$ & $10(11 \%)$ \\
\hline $4-7$ & $29(32 \%)$ \\
\hline $7-14$ & $52(57 \%)$ \\
\hline total & 91 \\
\hline
\end{tabular}

Fig.1 Demographic profile

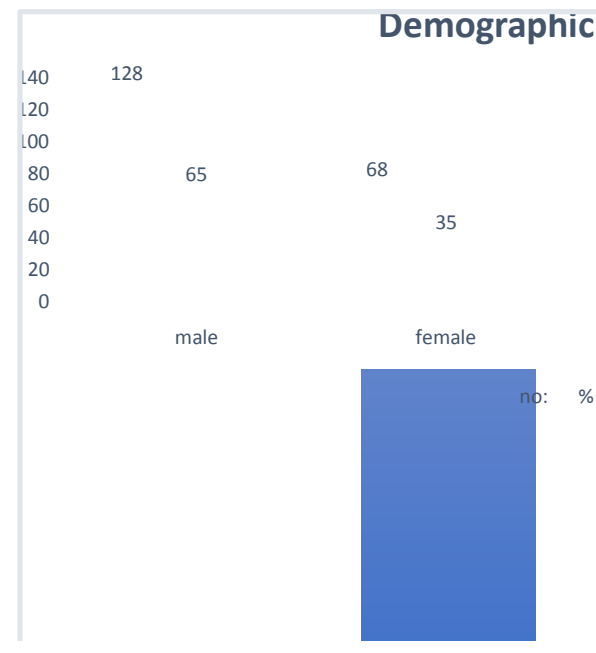

Fig.2 Age distribution

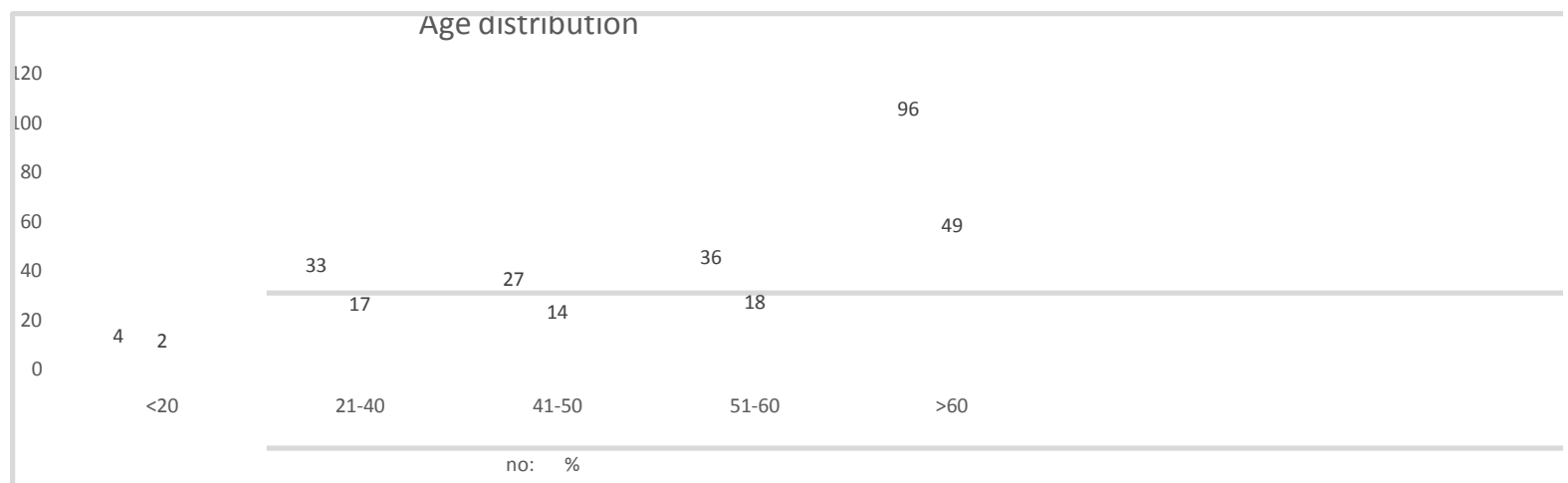


Fig.3 Urine culture showing positivity

Urine culture showing positivity

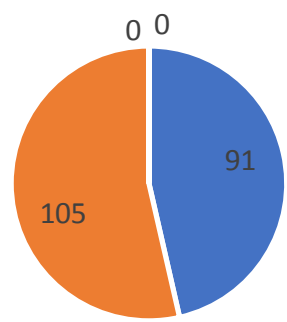

- growth no growth

Fig.4 Distribution of Bacterial isolates

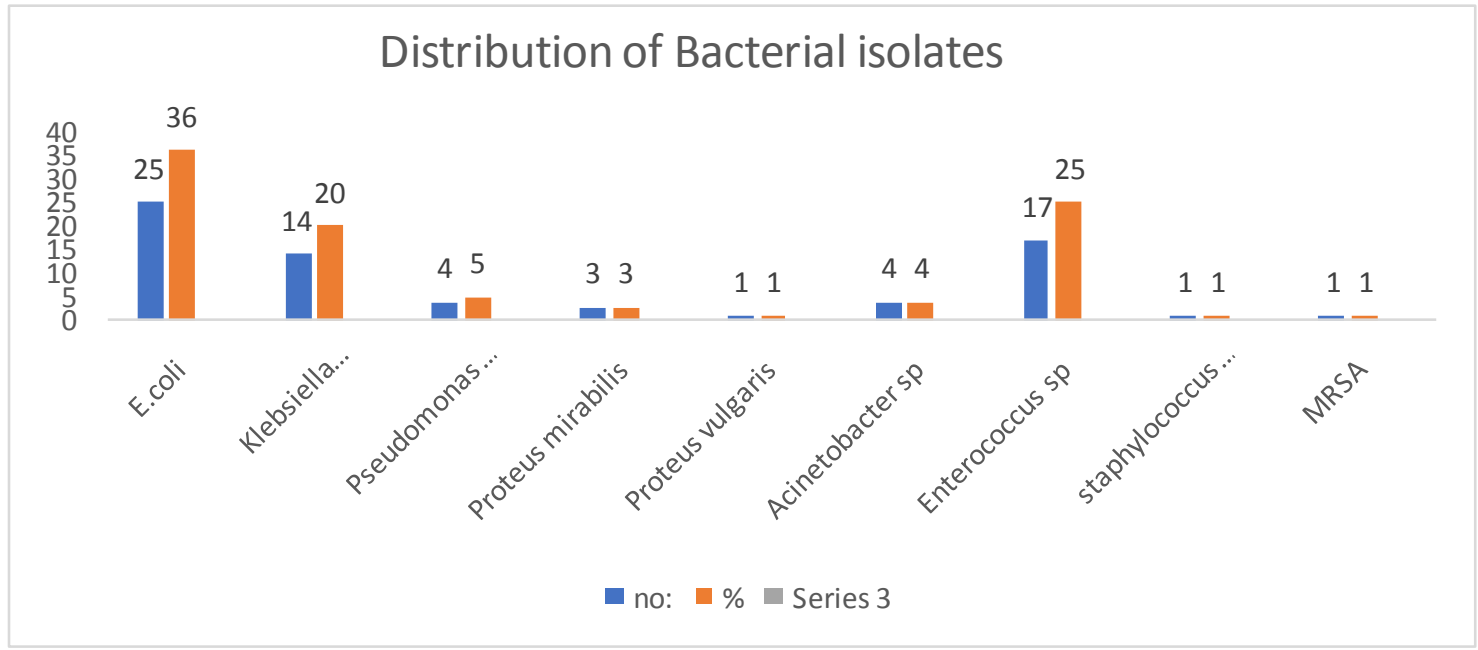

Fig.5 Distribution of fungal isolates 
Urinary catheter is inserted in more than 5 million patients in Emergency critical care hospital settings and extended care facilities. Therefore, these are at increased risk for CAUTI and its related sequelae. Worldwide per urethral catheter is identified as single most important predisposing factor for UTI. Catheter may serve as portal of entry for the pathogen if not aseptically inserted (Barbara et al., 2004; Ihnsook Jeong et al., 2010). In healthy patients, catheter associated colonization is usually asymptomatic, which resolves spontaneously after the removal of the catheter. In susceptible patients, colonization persists and leads infection. The complication of which could be such as prostatitis, epididymitis, cystitis, pyelonephritis and septicemia due to Gramnegative bacteremia particularly in high-risk patients (Chanda et al., 2015).

The etiology of UTI is varied. Infection is caused by a variety of pathogens including $E$. coli, Klebsiella, Proteus, Enterococci, Pseudomonas, Enterobacter and Candida. Many of these pathogens are part of patients own flora but can be acquired by cross contamination from other patients or hospital personnel or by exposure to contaminated solutions or nonsterile equipment (Singh et al., 2014).

Further CAUTI is most common cause of nosocomial infection (Naveen et al., 2016; Singh et al., 2010; Hooton et al., 2010). It also extends the hospital stay and adds to the direct cost of acute care hospitalization. It is associated with increased mortality. Study by Platt et al., (1982) and Kunin et al., (1992) suggested that nosocomial CAUTI are associated with substantially increased institutional death rates (15. Sanjay Saintet Jennifer et al., 2010).

In our study among the catheterized patients in ICU, infection found in $46 \%$ of patients, this is low as compared to the Karina et al.,
(1999) study, Hooton et al., (2010) which recorded incidence of catheter-related UTI as $51.4 \%$ while it is comparable with study by Mulhall et al., (1988) which reported a 44\% incidence Hooton et al., (2010).

Several risk factors have been cited to be associated with catheter-related UTI; these include advanced age, debilitation, postpartum state, etc., (Hooton et al., 2010). As advancing age is one of the predisposing host factor for development of CAUTI, the largest age group in our study included $>60$ years of age $(49 \%)$ while $17 \%$ of the patients were between 50 - 60 years of age (Fig. 2). Female gender is another significantrisk factor, concurring with the result of other investigators. The increased risk among women is probably due to anatomic makeup, causing an easier access of the perineal flora to the bladder along the catheter as it traverses the shorter female urethra (Hooton et al., 2010). However, in our study males were predominantly affected. This might be due to multiple factors. A higher number of male samples as compared to female samples received and male are prone to obstructive urinary lesion especially from benign prostate hypertrophy, $\mathrm{Ca}$ prostate and stricture associated with advanced age.

Prolonged catheterization is one of the significant risk factors for the development of UTI (Hooton et al., 2010). In the present study, the rate of development of UTI was higher as the duration of catheterization increased. This is similar to the Taiwo and Aderounmu study (2006) and Hooton et al., (2010).

The most common bacterial pathogen isolated in our study was E. coli 25 (36\%), followed by Klebsiella spp. 14 (20\%), Pseudomonas spp. 4 (5\%). Among the Gram-positive pathogens, Group D Streptococci (Enterococci) 17 (25\%) found to be most 
common, followed by Methicillin-resistant $S$. aureus $1(1 \%)$.

In a study by Taiwo and Aderounmu (2006) Klebsiella spp. (36\%) were the most common pathogen, followed by Pseudomonas (27\%), E. coli $(20 \%)$, S. aureus (10\%), Proteus mirabilis (3\%), C. albicans (3\%) and CONS as $(1.6 \%)$. Most of the studies like Selden et al., (1971); Oni et al., (2003) found the similar pattern of isolation (Hooton et al., 2010).

However, study by Karina et al., (1999) found a similar pattern like the present study. They found $E$. coli the most common pathogen (27) followed by Klebsiella spp. (26\%), Pseudomonas (8\%). Among the Grampositive organisms, Enterococcus (9\%), followed by CONS and $S$. aureus were reported by that study (Hooton et al., 2010).

In vitro antibiotic susceptibility pattern of the isolates showed the high level of resistance to commonly used drugs for UTI such as gentamicin (62\%), norfloxacin (68\%), nalidixic acid $(81.1 \%)$, ceftazidime $(65.5 \%)$, cefotaxime $(70 \%)$ and amikacin $(75 \%)$. The highest resistance was seen among the Klebsiella isolates (nalidixic acid-86\%, cefotaxime-86\%). The high resistance rate among the isolates observed in our study may be part due to the design of our study as it involved patients from ICUs. These patients generally undergo various empiric antimicrobial regimens and are, therefore, prone to develop infections by resistant pathogens.

Candidial infections of urinary tract are strongly associated with urinary catheterization (Ihnsook Jeong et al., 2010). In our study, non-albicans Candida found to be more common than $C$. albicans

In conclusion the urinary tract of catheterized patients is highly susceptible to severe infection. This infection is associated varied microbiological etiology. Antibiotic sensitivity pattern of the pathogen involved is also low. This along with existing underlying condition increases hospitalization, medication, morbidity and also adds to the financial burden. Therefore, it is imperative to carry out microbiological testing to determine etiology and ascertain effective antibiotics. Emphasis should also be made on reducing the duration of catheterization in order to reduce the incidence of catheter-related UTI. Hospital-wide surveillance program and appropriate catheter care protocols should be developed and implemented from evidencebased protocol.

\section{References}

Bagchi, et al., 2015. Microbiological Evaluation of Catheter Associated Urinary Tract Infection in a Tertiary Care Hospital. People's J. Sci. Res., $8(2)$

Barbara W. Trautner, Rabih O. and Darouiche. Catheter-Associated InfectionsArch Intern Med. 2004 April 26; 164(8): 842-850

Chanda R. Vyawahare, Nageswari R. Gandham, Rabindra Nath Misra et al., 2015. Occurrence of catheterassociated urinary tract infection in critical care units. Med. J. D.Y. Patil Univ., 8(5).

Ihnsook Jeong, R.N., Soonmi Park, M.S.N., Jae Sim Jeong, R.N. et al., 2010. Comparison of catheter-associated urinary tract infection rates by perineal care agents in intensive care units. Asian Nurs. Res., 4(3).

Karina, B.D., Myrna, M.T. and Tessa, T.T. 1999. Catheter related Urinary tract infections: Incidence, risk factors and microbiologic profile. Phil. J. Microbiol. Infect. Dis., 28: 133-8. 
Kunin, C.M., Douthitt, S., Dancing, J., Anderson, J., Moeschberger, M. 1992. The association between the use of urinary catheters and morbidity and mortality among elderly patients in nursing homes. Am. J. Epidemiol., 135: 291-301.

Mulhall, A.B., Chapman, R.G., Crow, R.A. 1988. Bacteriuruia during indwelling urethral catheterisation. J. Hosp. Infect., 11: 253-62.

Naveen, G., Nagraj, C., Latha, G. et al., 2016. Bacteriological Study of Catheter Associated Urinary Tract Infection in a Tertiary Care Hospital. Int. J. Curr. Microbiol. App. Sci., 5(9): 640-644

Oni, A.A., Mbah, G.A., Ogunkunle, M.O., Shittu, O.B., Bakare, R.A. 2003. Nosocomial infection: Urinary tract infection in patient with indwelling urinary catheter. Afr. J. Clin. Exp. Microbiol., 4: 63-71.

Platt, R., Polk, B.F., Murdock, B. and Rosner, B. Mortality associated with nosocomial urinary-tract infection. $N$. Engl. J. Med., 307: 637-41.

Sanjay Saintet Jennifer A. Meddings, MD2,3, David Calfee, et al., Catheterassociated Urinary Tract Infection and the Medicare Rule Changes Ann Intern Med. Author manuscript; available in PMC 2010 June 16
Selden, R., Lee, S., Wang, W.L., Bennett, J.V., Eickhoff, T.C. 1971. Nosocomial Klebsiella infections: Intestinal colonization as a reservoir. Ann. Intern. Med., 74: 657-64.

Singh, M. et al., 2014. Per Urethral Catheterisation: Microbial Growth Incidence and its Management Indian. J. Pharm. Biol. Res., 2014; 2(3): 105111.

Singh, S., Pandya, Y., Patel, R., Paliwal, M. et al., 2010. Surveillance of deviceassociated infections at a teaching hospital in rural Gujarat. Indiaijmm., 28(4): 342-7.

Taiwo, S.S., Aderounmu, A.O.A. 2006. Catheter associated urinary tract infection: Aetiologic agents and antimicrobial susceptibility pattern in Ladoke Akintola University Teaching Hospital, Osogbo, Nigeria. Afr. $J$. Biomed. Res., 9: 141-148.

Thomas M. Hooton, Suzanne F. Bradley et al., 2010. Diagnosis, Prevention, and Treatment of Catheter Associated Urinary Tract Infection in Adults: 2009 International Clinical Practice Guidelines from the Infectious Diseases Society of America CID 2010:50.

\section{How to cite this article:}

Sangamithra, V., Sneka, Shabana Praveen and Manonmoney. 2017. Incidence of Catheter Associated Urinary Tract Infection in Medical ICU in a Tertiary Care Hospital. Int.J.Curr.Microbiol.App.Sci. 6(4): 662-669. doi: https://doi.org/10.20546/ijcmas.2017.604.081 